Since September 1961, weather observations were made in Jiri, East No. 2, Nepal, through the Geographical Institute of the University of Zurich in cooperation with SHAG (Schweizerisches Hilfswerk für außereuropäische Gebiete). The results of the first two years (September 1961 through July 1963) are published in table I and figure 1. Another station, in the vicinity of Jiri at $3000 \mathrm{~m}$, Thodung, operated during the same period to determine the vertical temperature gradient. Because of local circumstances the Thodung station did not give a uninterrupted record (table II), but for the purpose mentioned above the data collected were sufficient (table III). The present paper simply presents the data and reports on the operational aspects.

\title{
EINE BALINESISCHE STOFFMALEREI MIT MYTHOLOGISCHER SZENE
}

\author{
ALFRED STEINMANN
}

Mit einem Bild

$\mathrm{Zu}$ den kulturgeschichtlich und ethnologisch interessanten Zeugnissen indonesischer Kunst gehören zweifellos die in den Beständen der meisten völkerkundlichen Museen sowie mancher Privatsammlungen anzutreffenden Malereien auf Baumwollstoff aus der Insel Bali. Auch vom rein künstlerischen Standpunkt aus nehmen sie durch ihre besondere, durch bestimmte Stilmerkmale gekennzeichnete und durch Tradition vorgeschriebene stereotype Darstellungsart einen einzigartigen Platz ein. Unter den nach Größe und Format recht verschiedenen Exemplaren unterscheidet man quadratische Stücke (ulun-ulun), rechteckige Formen (tabing) sowie lange und schmale Streifen (iderider), ähnlich denjenigen, die früher vor den Zuschauern des alten Wayang-beberTheaters abgerollt wurden. Abgesehen von den zu horoskopischen und anderweitigen Wahrsagezwecken dienenden astrologischen Bildkalendern ${ }^{1}$ (palalintangan), liegen den balinesischen Stoffmalereien (es sind eigentlich eher mit Wasserfarben kolorierte Zeichnungen ${ }^{2}$ ) in der Regel Erzählungen aus dem indischen Sagenkreis, zum Teil in ihrer altjavanischen Bearbeitung zugrunde. Meistens werden sie zur Verzierung von Wohnräumen verwendet.

Für den Laien ist es oft recht schwierig, die dargestellten Szenen zu deuten und ihren Sinn zu verstehen. Aber auch für den Fachmann gibt es neben leicht deutbaren Darstellungen oft solche, die nur durch Heranziehung der ihnen zugrunde liegenden Texte und durch Vergleich mit ihnen interpretiert werden können. $\mathrm{Zu}$ den beliebten Themen gehören unter anderem Szenen, die sich auf die Episode der Quirlung des Milchmeeres durch Götter und Dämonen und auf diejenige des nachfolgenden Kampfes um den begehrten Unsterblichkeitstrank (Amrita) beziehen ${ }^{3}$. Im Besitz der Zürcher

1 Siehe u. a. A. Steinmann: Ein astrologischer Wahrsagekalender aus Bali. (Blätter für die Kunst, Zürich, Nr.4, Juli 1941).

2 Als solche wurden sie schon früher durch H. H. Juynboll: Proeve van balines. teekenkunst, (I. A. E. Bd. 16, 1904, S. 81) und durch P.V. van Stein-Callenfels: De Mintaraga-basreliefs aan de Oudjavaansche bouwwerken. (Publ. v/d Oudheidk. Diesnt in Ned. Indië, deel 1, 1925, S. 52) bezeichnet, weil alle Objekte feine schwarze Umrißlinien besitzen und der Farbengebung dagegen eine eher untergeordnete Bedeutung zuzukommen scheint.

3 Die letztgenannte Episode ist auf einer im Institut Kern in Leiden vorhandenen balinesischen «Malerei» wiedergegeben, die von A. J. Goedheer: De strijd om de onsterfelijkheidsdrank op een Balisch doek (Cultureel Indië, 1. jaarg. 1939, S. $344-46$ ) abgebildet und gedeutet worden ist. 
Universitätssammlung für Völkerkunde befindet sich unter anderen ein großformatiges Stoffgemälde vom «tabing»-Typus $(255 \mathrm{~cm} \times 132 \mathrm{~cm})$ mit einer solchen Darstellung4 (siehe Bild). Diese im Schrifttum der Inder in verschiedenen Versionen verbreitete und auch auf Tempel-Basreliefs abgebildete Episode ${ }^{5}$ ist den Balinesen aus der altjavanischen Bearbeitung des ersten Teils des im gesamten indischen Kulturgebiet (einschließlich Indonesiens) bekannten und beliebten Heldenepos Mâhabhârata des sog. «Adiparwa», geläufig und ein bevorzugtes 'Thema für die künstlerische Gestaltung durch ihre Zeichner und Maler geworden.

Zum besseren Verständnis der nachfolgenden Bildinterpretation geben wir hier vorgängig eine kurze Zusammenfassung des Inhalts der betreffenden Mythe:

Dem Ratschlag des Gottes Vishnu Folge leistend, haben die Götter und Dämonen (asuras) miteinander vereinbart, gemeinsam das Milchmeer zu quirlen, um in den Besitz des Unsterblichkeitstrankes sowie anderer Kostbarkeiten zu gelangen. Als Quirlstab oder Quirlstock für das Umbuttern dient der (mit Hilfe des Schlangenfürsten Ananta aus der Erde gezogene) Berg Mandara, während die um ihn gerollte Schlange Vâsuki als Quirlstrick benützt wird. Beim Quirlen werden die Götter an das Schwanzende, die Dämonen dagegen beim Kopfteil der Schlange postiert, was sich wegen des giftigen dem Schlangenrachen entströmenden Flammenhauches für sie störend auswirkt, während umgekehrt die am anderen Ende befindlichen Götter durch liebliche Wolken gekühlt und erfrischt werden. Vishnu, der sich für dieses von ihm vorgeschlagene Vorhaben in verschiedener Gestalt einsetzt, taucht in seiner Verkörperung als Riesenschildkröte (Kûrma) auf den Meeresgrund, um mit seinem Rücken dem Berg Mandara als Basis zu dienen. Zudem überwacht er in seiner normalen göttlichen Gestalt den Quirlvorgang und hilft sogar persönlich mit. Nach langem Quirlen kommen allerlei wunderbare Kostbarkeiten ${ }^{6}$ und darunter endlich auch der Unsterblichkeitstrank zum Vorschein. Zunächst vermögen sich die Dämonen des Wundertrankes zu bemächtigen, indem sie die Schale den Händen Dhavantaris entreißen, doch gelingt es Vishnu, diesmal in der Gestalt einer verführerischen Frau, die Dämonen derart abzulenken, daß sie ihm das Gefäß mit dem Nektar überlassen. Mit der erneuten Inbesitznahme des Trankes durch die Götterschar beginnt die zweite, hier nicht mehr abgebildete ${ }^{7}$ Episode des im Schrifttum ausführlich geschilderten Kampfes zwischen den beiden Parteien, der schließlich mit der endgültigen Niederlage und dem Rückzug der Dämonen endigt.

Auf der vorliegenden Malerei lassen sich die wesentlichen Elemente der oben genannten Mythe unschwer erkennen. Das Umbuttern des Milchozeans ist hier als Hauptvorgang recht anschaulich dargestellt. In der Bildmitte erhebt sich der massive, aus den Fluten emporsteigende und von dem als riesige Meeresschildkröte erscheinenden Gott Vishnu getragene Berg Mandara, um den sich die als Quirlstab fungierende Schlange Vâsuki windet. An ihrem hinteren Teil (links auf dem Bild) erblicken wir die

4 Eine ähnliche Szene war übrigens seinerzeit auf einer balinesischen Malerei im «Musée d'Ethnographie de Neuchâtel» anläßlich der Ausstellung «Indonésie, les Iles des dieux». Nov. 1956 bis Februar 1957 zu sehen (vergl. Katalog, Section III, peintures traditionnelles, no. 2, S. 26 unten).

5 Diesbezügliche Darstellungen kennt man u. a. auf den Reliefs von Conjeeveram (siehe E. Abegg: Die Indiensammlung der Univ. Zürich, 1934, S. 4o), ferner auf denjenigen der Khmertempel in Cambodja. Bezüglich des Schriftums sei auf die Sammlung vishnuitischer Mythen, den Bhâgavata Purâna aus dem 7. Jahrh. verwiesen.

6 Unter denen beiläufig der Mond, das weiße Pferd Uccaihshravas und der weiße Elefant Airâvata, die Wunschkuh Kâmadhênu, Vishnus Gemahlin Lakshmi, ferner die Göttin des Weines Sura, die schöne Nymphe Rambhâ, Vishnus Juwel Kaustubha, das Gift Kâlakuta, (das die Welt zu vernichten droht, jedoch auf Brahma's Geheiß durch Shiva zur Rettung der Welt vor dem Untergang verschluckt wird) und unter anderem endlich auch der vielbegehrte, in einem Gefäß schäumende Unsterblichkeitstrank, das der schwarze Jüngling und Götterarzt Dhavantari in den Händen trägt.

7 Aber u. a. auf der Leidener Balimalerei anzutreffende 


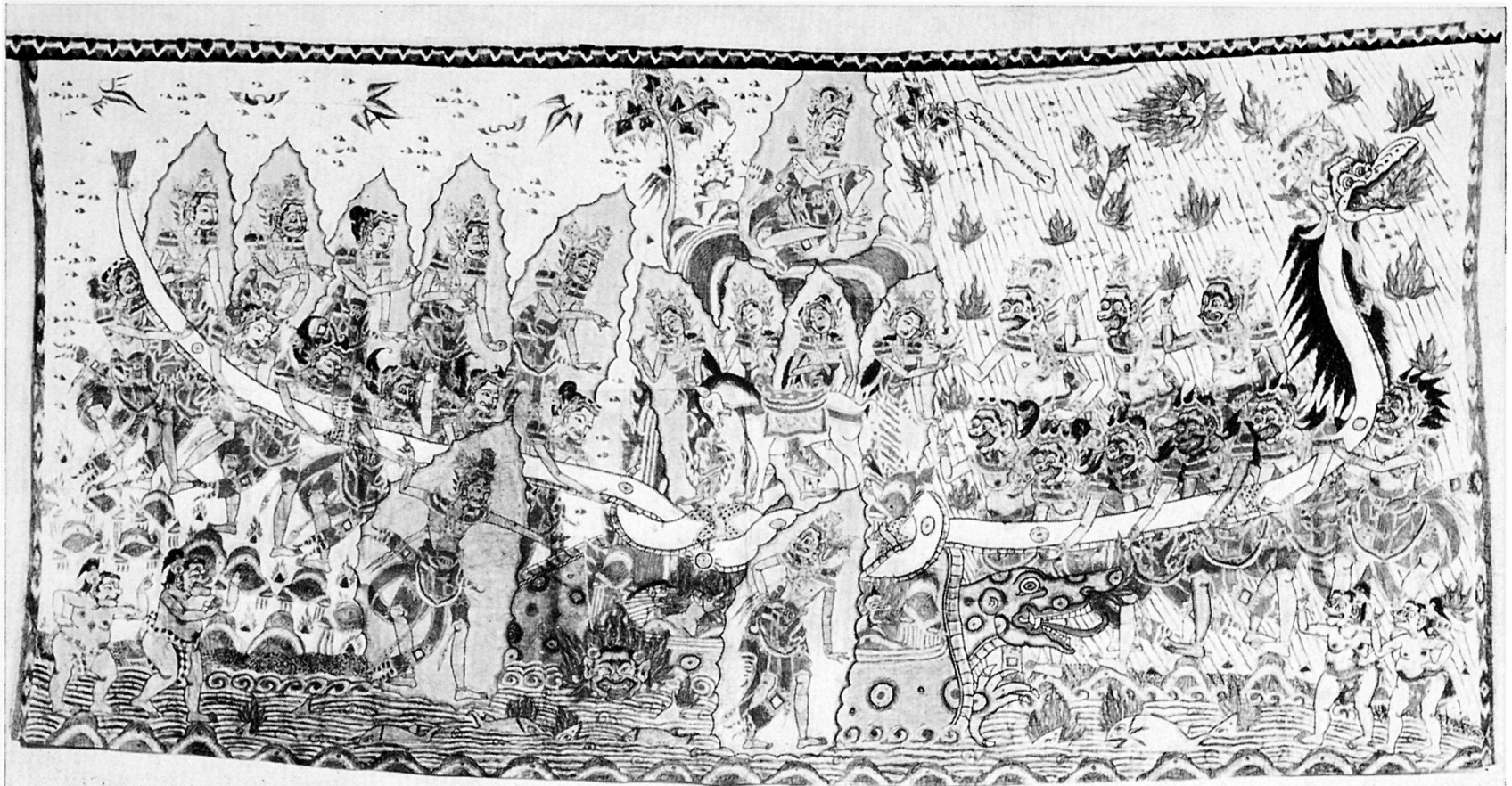

Die Quirlung des Milchozeans zur Erlangung des Unsterblichkeitstrankes.

(Malerei auf Baumwolle, Bali. $255 \mathrm{~cm}$ x $132 \mathrm{~cm}$.) Sammlung für Völkerkunde der Universität Zürich. 
jeweils von einer Aureole umrahmten Gottheiten, denen rechts, am Kopfteil der Schlange, die Dämonenschar gegenübersteht. Eindrücklich hat der Künstler die aus dem Schlangenrachen kommenden und den Luftraum um die Dämonen vergiftenden Flammen zur Darstellung gebracht, die unter anderem den Baum rechts auf dem MandaraBerg bereits angesengt und die zahlreichen auf dem Meere treibenden Fische verbrannt haben. Auf der Götterseite erscheint der Himmel dagegen klar und mit Wölkchen und Vögeln belebt. Von den zahlreichen in der indischen Mythe erwähnten Kostbarkeiten, die aus dem gekarnten Milchmeer zum Vorschein kommen, sind hier (vielleicht um das Gesamtbild nicht allzusehr zu überlasten und zu verwirren) nur wenige dargestellt: in erster Linie das an zentraler Stelle stehende weiße Pferd Uccaihshravas und darüber vier weibliche göttliche Wesen mit Aureolen, unter denen wir links Vishnus Gattin Lakshmi mit der Lotusknospe in der Hand erkennen. Bei der zweiten und dritten dürfte es sich um die Göttin Sura und die Nymphe Rambhâ handeln. Die vierte weibliche Gestalt rechts, welche ein von einem Dämon dargebotenes Gefäß in Empfang nimmt, kann wohl niemand anderes sein als Vishnu selbst, diesmal als betörende weibliche Erscheinung, der ein Dämon die Schale mit dem Wundertrank überreicht ${ }^{8}$. Immer als Hauptperson wird auch hier, wie auf einem Tempelrelief von Angkor-Vat ${ }^{9}$, Vishnu verschiedene Male abgebildet : zunächst auf dem Gipfel des Berges in Sitzpose thronend und den Quirlungsverlauf überwachend, ferner als dunkelhäutige Göttergestalt zweimal links von der Mitte, wo er als Anführer der Götterschar mit bezeichnender Gebärde aktiv am Quirlvorgang sich beteiligt, und nochmals etwas weiter unten, wo er in einer nicht näher festzustellenden Auseinandersetzung mit einer anderen Gottheit begriffen ist, und endlich, wie bereits erwähnt, in tierischer Erscheinungsform als Riesenschildkröte im Meer, die vom Künstler offenbar recht frei und phantasievoll gestaltet ist und eher an den Fischelefant Makara erinnert. Unten auf dem Bild betrachten rechts und links die bekannten und beim indonesischen Volk so beliebten Spaßmacher und Diener der Götter, die Panakawan, die Vorgänge.

Die Bildinhalte der vorliegenden Bali-Malerei und des Leidener Exemplars (das von Goedheer publizierte Bild ist zur Reproduktion ungeeignet, so daß wir es hier nicht zeigen können) ergänzen einander. Zusammen geben sie ein geschlossenes Bild der hier besprochenen Mythe.

\section{INTERPRÉTATION D'UNE PEINTURE TRADITIONNELLE BALINAISE SUR TOILE}

Il s'agit de l'épisode du barattement de l'océan de lait (dont fait partie l'avatar de Vishnou sous la forme d'une tortue) en vue de se procurer l'amrita, élixir tant convoité de l'immortalité. Cette épisode, qui est devenue aussi populaire au Cambodge que dans l'Inde, et dont les scènes ont été reproduites par les sculpteurs sur les bas-reliefs des temples d'Angkor-Vat et de Conjeeveram, se trouve entre autre dans un recueil de mythes vishnouites, le Bhâgavata-Pourana, rédigé vers le VIIe siècle apr. J. Ch. En Indonésie et notamment à Bali, l'épisode est connue sous son ancienne version javanaise de la première partie du poème Mahabharata, l'«Adiparwa».

De ce barattage, pour lequel les dieux et les démons (asouras) utilisent le serpent Vasouki en guise de corde, enroulé autour du mont Mandara servant de pivot, sortirent d'abord quantité de trésors et d'être fabuleux (entre autre le cheval blanc Uccaihshravas), puis, enfin, la coupe contenant le breuvage de l'immortalité dont les démons s'emparèrent. Il en suit un combat acharné qui se termine par l'apparition de Vishnou sous les traits d'une séduisante femme, dont la beauté détourna leur attention et qui put ainsi restituer l'élixir aux dieux. La scène du combat, qui ne figure plus sur notre peinture, est reproduite sur une autre peinture qui se trouve à l'institut Kern de Lyde, publiée et illustrée en 1939 par H. H. Goedheer.

8 Derselbe Vorgang ist in ähnlicher, ja fast identischer Darstellung auf der bereits erwähnten Bali-Malerei in Leiden zu sehen, die aber nicht die Quirlung des Meeres, sondern den Endkampf zwischen Göttern und Asuras zum Hauptthema hat.

9 Siehe Abbildung 21 auf S. 195 in der «Mythologie asiatique illustrée», Paris, Librairie de France, 1928. 